\title{
$K_{l 3}$ form factor with two-flavors of dynamical domain-wall quarks
}

\section{RBC Collaboration:}

\section{Dawson}

RIKEN-BNL Research Center, Brookhaven National Laboratory, Upton, NY 11973, USA

\section{T. Izubuchi}

Institute of Theoretical Physics, Kanazawa University, Kanazawa 920-1192, Japan

RIKEN-BNL Research Center, Brookhaven National Laboratory, Upton, NY 11973, USA

\section{T. Kaneko*}

High Energy Accelerator Research Organization (KEK), Ibaraki 305-0801, Japan

The Graduate University for Advanced Studies, Ibaraki 305-0801, Japan

\section{S. Sasaki}

Department of Physics, University of Tokyo, Tokyo 113-0033, Japan

RIKEN-BNL Research Center, Brookhaven National Laboratory, Upton, NY 11973, USA

\section{A. Soni}

Physics Department, Brookhaven National Laboratory, Upton, NY 11973, USA

We report on our calculation of $K \rightarrow \pi$ vector form factor by numerical simulations of two-flavor QCD on a $16^{3} \times 32 \times 12$ lattice at $a \simeq 0.12 \mathrm{fm}$ using domain-wall quarks and DBW2 glue. Our preliminary result at a single sea quark mass correponding to $m_{\mathrm{PS}} / m_{\mathrm{V}} \simeq 0.53$ shows a good agreement with previous estimate in quenched QCD and that from a phenomenological model.

XXIIIrd International Symposium on Lattice Field Theory

25-30 July 2005

Trinity College, Dublin, Ireland

\footnotetext{
*Speaker.
} 


\section{Introduction}

Currently, $K \rightarrow \pi l v_{l}\left(K_{l 3}\right)$ decay provides the most precise determination of the CabibboKobayashi-Maskawa (CKM) matrix element $\left|V_{u s}\right|$ through $\Gamma \propto\left|V_{u s}\right|^{2}\left|f_{+}(0)\right|^{2}$, where $\Gamma$ is the decay rate and $f_{+}\left(q^{2}\right)$ is the form factor defined from the $K \rightarrow \pi$ matrix element of the weak vector current

$$
\left\langle\pi\left(p^{\prime}\right)\left|V_{\mu}\right| K(p)\right\rangle=\left(p_{\mu}+p_{\mu}^{\prime}\right) f_{+}\left(q^{2}\right)+\left(p_{\mu}-p_{\mu}^{\prime}\right) f_{-}\left(q^{2}\right), \quad q^{2}=\left(p-p^{\prime}\right)^{2} .
$$

While the PDG value for the CKM matrix elements [1] shows a $2 \sigma$ deviation from the CKM unitarity

$$
\left|V_{u d}\right|^{2}+\left|V_{u s}\right|^{2}+\left|V_{u b}\right|^{2}=1-\delta, \quad \delta=0.0033(15),
$$

recent experiments for $\Gamma[2,3,4,5]$ prefer a slightly larger value of $\left|V_{u s}\right|$, which is consistent with the unitarity $(\delta=0)$. However, in order to make a definite conclusion on this issue, $f_{+}(0)$ has to be determined theoretically with an accuracy of about $1 \%$.

In chiral perturbation theory (ChPT), $f_{+}(0)$ is expanded in powers of meson masses $M_{K}, M_{\pi}$, and $M_{\eta}$

$$
f_{+}(0)=1+f_{2}+f_{4}+\cdots, \quad\left(f_{n}=O\left(M_{K, \pi, \eta}^{n}\right)\right) .
$$

Thanks to the Ademollo-Gatto theorem [6], which states that $S U(3)$ breaking effects in $f_{+}(0)$ start at $O\left(\left(m_{s}-m_{u d}\right)^{2}\right)$, poorly-known low-energy constants of the chiral Lagrangian do not enter the ChPT formula for $f_{2}$ [7], and hence it is precisely determined as -0.023 . However, this is not the case for the higher order corrections $f_{n}(n \geq 4)$ [8]. Therefore, a phenomenological estimate of $f_{4}$ based on the quark model [9] has been used in previous analyses of $\left|V_{u s}\right|$.

In order to estimate $f_{+}(0)$ without relying on any phenomenological model, lattice calculations have been carried out first in the quenched approximation [10], and later in unquenched QCD [11, 12]. In these calculations, however, conventional Wilson- or Kogut-Susskind-type quark actions are employed, and hence chiral properties of $f_{+}(0)$ may be significantly affected by the explicit breaking of chiral or flavor symmetry. Since the ChPT formula for $f_{2}$ plays a crucial role in the chiral extrapolation of lattice data, it is advantageous to use a quark action, which posses chiral symmetry even at finite lattice spacing. In this work, we calculate $f_{+}(0)$ in two-flavor dynamical QCD using the DBW2 gauge [14] and the domain-wall quark actions [13], with which the hadron spectrum and the kaon $B$ parameter show good chiral properties [15].

\section{Simulation method}

Our calculations are carried out on a $16^{3} \times 32$ lattice with statistics of $4750 \mathrm{HMC}$ trajectories. While we simulate a single value for the lattice spacing $a^{-1}=1.69(5) \mathrm{GeV}$, we expect that the scaling violation in $f_{+}$is not large, since the employed lattice action is (automatically) $O(a)$-improved. The size of the fifth dimension is set to $L_{5}=12$, which leads to the residual mass of a few MeV. We refer to Ref. [15] for details on the gauge ensembles used in this study.

At the moment, we complete our calculation at a single sea quark mass $m_{u d}=0.02$, which is roughly half the physical strange quark mass. Three heavier masses $m_{\mathrm{s}}=0.03,0.04$ and 0.05 are employed for the valence strange quarks. 


\section{Extraction of form factor}

The so-called scalar form factor

$$
f_{0}\left(q^{2}\right)=f_{+}\left(q^{2}\right)+\frac{q^{2}}{M_{K}^{2}-M_{\pi}^{2}} f_{-}\left(q^{2}\right)
$$

at $q_{\max }^{2}=\left(M_{K}-M_{\pi}\right)^{2}$ can be extracted from the double ratio proposed in Ref. [16],

$$
\begin{aligned}
R\left(t, t^{\prime}\right) & =\frac{C_{4}^{K \pi}\left(t, t^{\prime} ; \mathbf{0}, \mathbf{0}\right) C_{4}^{\pi K}\left(t, t^{\prime} ; \mathbf{0}, \mathbf{0}\right)}{C_{4}^{K K}\left(t, t^{\prime} ; \mathbf{0}, \mathbf{0}\right) C_{4}^{\pi \pi}\left(t, t^{\prime} ; \mathbf{0}, \mathbf{0}\right)} \underset{t,\left(t^{\prime}-t\right) \rightarrow \infty}{\longrightarrow} \frac{\left(M_{K}+M_{\pi}\right)^{2}}{4 M_{K} M_{\pi}}\left|f_{0}\left(q_{\max }^{2}\right)\right|^{2}, \\
C_{\mu}^{P Q}\left(t, t^{\prime} ; \mathbf{p}, \mathbf{p}^{\prime}\right) & =\sum_{\mathbf{x}, \mathbf{x}^{\prime}}\left\langle O_{Q}\left(\mathbf{x}^{\prime}, t^{\prime}\right) V_{\mu}(\mathbf{x}, t) O_{P}^{\dagger}(\mathbf{0}, 0)\right\rangle e^{-i \mathbf{p}^{\prime}\left(\mathbf{x}^{\prime}-\mathbf{x}\right)} e^{-i \mathbf{p x}}, \quad(P, Q=\pi \text { or } K),
\end{aligned}
$$

where $O_{\pi(K)}$ represents the interpolating operator for pion (kaon). As shown in Fig. 1, $f_{0}\left(q_{\max }^{2}\right)$ is determined with an accuracy of $\lesssim 0.1 \%$, since various uncertainties of the three-point function, such as the statistical fluctuation, are canceled at least partially in the ratio.

To study the $q^{2}$ dependence of the form factor, we calculate

$$
F\left(p, p^{\prime}\right)=\frac{f_{+}\left(q^{2}\right)}{f_{0}\left(q_{\max }^{2}\right)}\left(1+\frac{E_{K}(\mathbf{p})-E_{\pi}\left(\mathbf{p}^{\prime}\right)}{E_{K}(\mathbf{p})+E_{\pi}\left(\mathbf{p}^{\prime}\right)} \xi\left(q^{2}\right)\right), \quad \xi\left(q^{2}\right)=f_{-}\left(q^{2}\right) / f_{+}\left(q^{2}\right)
$$

from a ratio

$$
\frac{C_{4}^{K \pi}\left(t, t^{\prime} ; \mathbf{p}, \mathbf{p}^{\prime}\right) C^{K}(t ; \mathbf{0}) C^{\pi}\left(t^{\prime}-t ; \mathbf{0}\right)}{C_{4}^{K \pi}\left(t, t^{\prime} ; \mathbf{0}, \mathbf{0}\right) C^{K}(t ; \mathbf{p}) C^{\pi}\left(t^{\prime}-t ; \mathbf{p}^{\prime}\right)} \underset{t,\left(t^{\prime}-t\right) \rightarrow \infty}{\longrightarrow} \frac{E_{K}(\mathbf{p})+E_{\pi}\left(\mathbf{p}^{\prime}\right)}{M_{K}+M_{\pi}} F\left(p, p^{\prime}\right),
$$

where $C^{\pi(K)}(t ; \mathbf{p})$ represents the pion (kaon) propagator with the spatial momentum $\mathbf{p}$. Figure 1 shows a plot of $F\left(p, p^{\prime}\right)$. The accuracy of $F\left(p, p^{\prime}\right)$ is typically $5-10 \%$ for the spatial momenta $|\mathbf{p}|,\left|\mathbf{p}^{\prime}\right| \leq \sqrt{2}$.

In order to convert $F\left(p, p^{\prime}\right)$ to $f_{0}\left(q^{2}\right), \xi\left(q^{2}\right)$ is estimated from the double ratio proposed in Ref.[10]

$$
\begin{aligned}
& R_{k}\left(t, t^{\prime} ; \mathbf{p}, \mathbf{p}^{\prime}\right)=\frac{C_{k}^{K \pi}\left(t, t^{\prime} ; \mathbf{p}, \mathbf{p}^{\prime}\right) C_{4}^{K K}\left(t, t^{\prime} ; \mathbf{p}, \mathbf{p}^{\prime}\right)}{C_{4}^{K \pi}\left(t, t^{\prime} ; \mathbf{p}, \mathbf{p}^{\prime}\right) C_{k}^{K K}\left(t, t^{\prime} ; \mathbf{p}, \mathbf{p}^{\prime}\right)} \quad(k=1,2,3), \\
& \xi\left(q^{2}\right)=\frac{-\left(E_{K}(\mathbf{p})+E_{K}\left(\mathbf{p}^{\prime}\right)\right)\left(p+p^{\prime}\right)_{k}+\left(E_{K}(\mathbf{p})+E_{\pi}\left(\mathbf{p}^{\prime}\right)\right)\left(p+p^{\prime}\right)_{k} R_{k}}{\left(E_{K}(\mathbf{p})+E_{K}\left(\mathbf{p}^{\prime}\right)\right)\left(p-p^{\prime}\right)_{k}-\left(E_{K}(\mathbf{p})-E_{\pi}\left(\mathbf{p}^{\prime}\right)\right)\left(p+p^{\prime}\right)_{k} R_{k}} .
\end{aligned}
$$
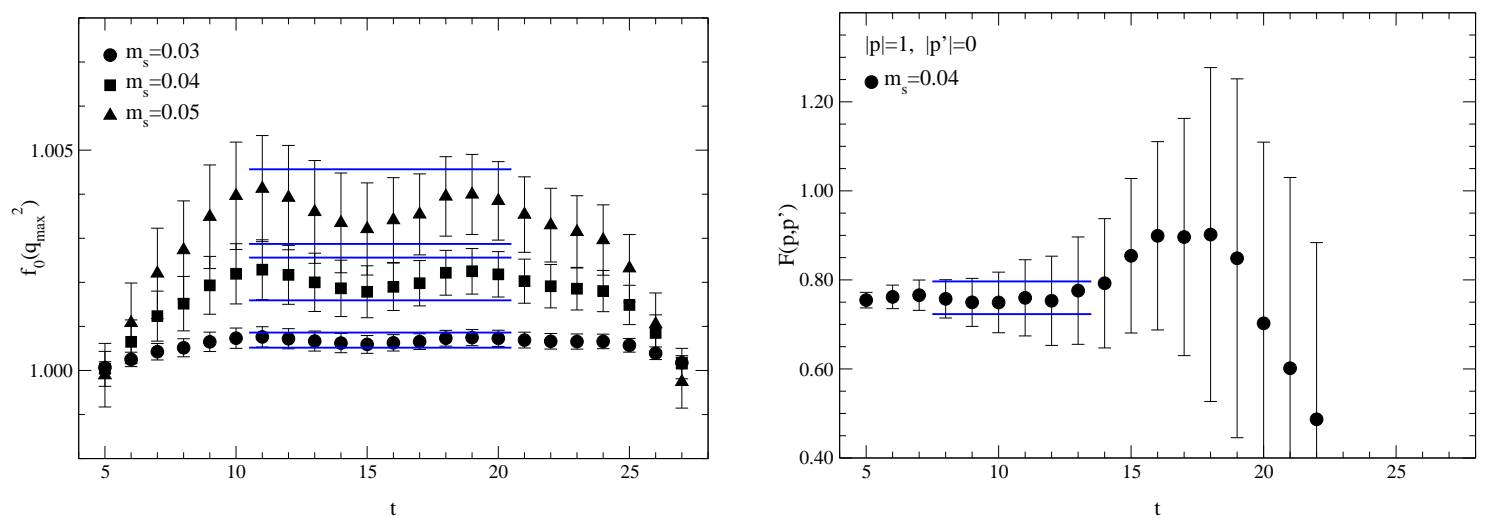

Figure 1: Plots of $f_{0}\left(q_{\max }^{2}\right)$ (left fi gure) and $F\left(p, p^{\prime}\right)$ with $|\mathbf{p}|=1$ and $\left|\mathbf{p}^{\prime}\right|=0$ (right fi gure) as a function of $t$. 
We observe that $\xi\left(q^{2}\right)$ has a mild dependence on the valence quark mass and its magnitude is typically $\simeq-0.01$ with $50-100 \%$ error.

\section{Interpolation to $q^{2}=0$}

Here, we test two methods to determine $f_{+}(0)\left(=f_{0}(0)\right)$.

- method-1: As in Ref.[10], we first calculate $f_{0}\left(q^{2}\right)$ at each simulated $q^{2}$ from $f_{0}\left(q_{\max }^{2}\right)$, $F\left(p, p^{\prime}\right)$ and $\xi\left(q^{2}\right)$, and then interpolate $f_{0}\left(q^{2}\right)$ to $q^{2}=0$.

- method-2: As in Ref.[11], $F\left(p, p^{\prime}\right)\left(\xi\left(q^{2}\right)\right)$ is interpolated (extrapolated) to $q^{2}=0$, and then $f_{0}(0)$ is calculated at $q^{2}=0$. The interpolation of $F\left(p, p^{\prime}\right)$ is carried out using data with fixed $|\mathbf{p}|$ (or $\left|\mathbf{p}^{\prime}\right|$ ) so that we can unambiguously identify $\left|\mathbf{p}^{\prime}\right|(|\mathbf{p}|)$ corresponding to $q^{2}=0$, which is needed to convert $\left.F\left(p, p^{\prime}\right)\right|_{q^{2}=0}$ to $f_{0}(0)$. We repeat this analysis for two data sets with $\mathbf{p}=\mathbf{0}$ and $\mathbf{p}^{\prime}=\mathbf{0}$, and take the average of results for $f_{0}(0)$.

We test quadratic and polar fits

$$
\begin{aligned}
& \mathscr{O}\left(q^{2}\right)=\mathscr{O}(0) \cdot\left(1+c_{1} q^{2}+c_{2} q^{4}\right), \\
& \mathscr{O}\left(q^{2}\right)=\frac{\mathscr{O}(0)}{1-c_{1} q^{2}}
\end{aligned}
$$

for the interpolation of $f_{0}$ in method-1 and $F$ for method-2, while only the quadratic fit is tested for $\xi$. We observe that four possible ways (quadratic or polar fit for method-1 or 2) give mutually consistent results with an accuracy of $\lesssim 1 \%$. This is because we have very accurate data of $f_{0}\left(q_{\max }^{2}\right)$ near $q^{2}=0$, and hence the uncertainty due to the choice of the interpolation method is not large.

However, we observe that method-2 leads to a slightly smaller error of $f_{0}(0)$ than method-1, since the kinematical factor $\left(E_{K}(\mathbf{p})-E_{\pi}\left(\mathbf{p}^{\prime}\right)\right) /\left(E_{K}(\mathbf{p})+E_{\pi}\left(\mathbf{p}^{\prime}\right)\right)$ in Eq. (3.4) is not large at $q^{2}=0$, and hence the uncertainty of $\xi(0)$ has small influence to $f_{0}(0)$ in method-2. Therefore, we employ $f_{+}(0)\left(=f_{0}(0)\right)$ obtained from method-2 with the quadratic fit in the following.
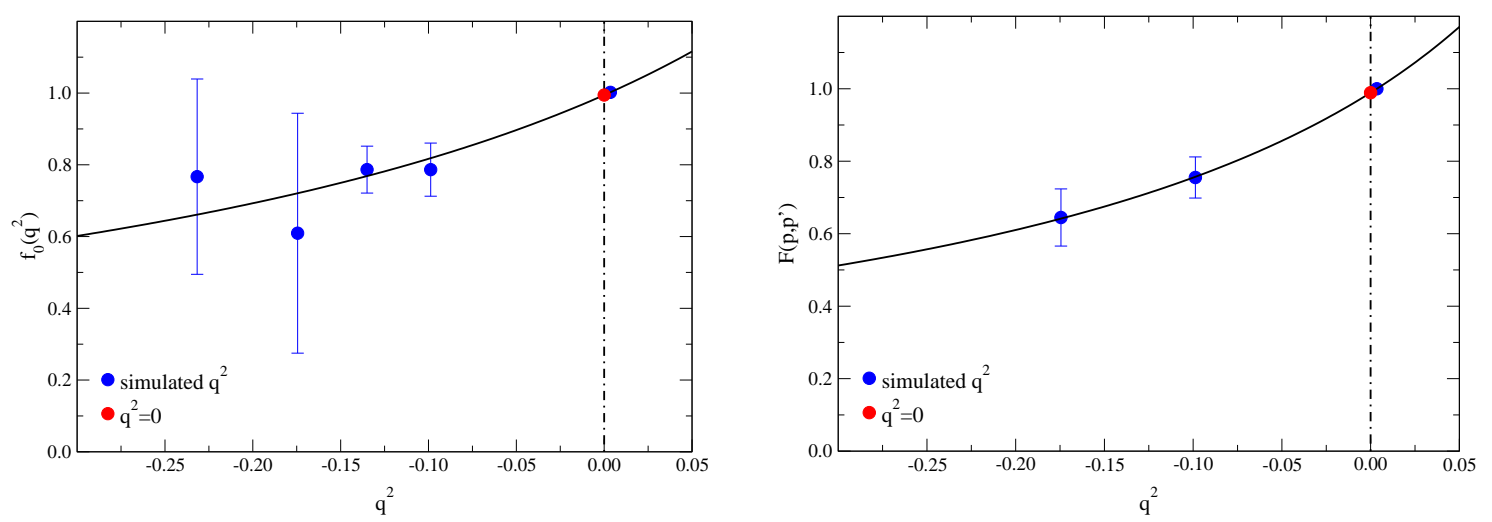

Figure 2: Interpolation of $f_{0}\left(q^{2}\right)$ (left fi gure) and $F\left(p, p^{\prime}\right)$ with $\mathbf{p}^{\prime}=\mathbf{0}$ (right fi gure) to $q^{2}=0$. Both fi gures show data at $m_{s}=0.04$. 


\section{Chiral extrapolation}

From the Ademollo-Gatto theorem, the higher order correction

$$
\Delta f=f_{+}(0)-\left(1+f_{2}\right)=\sum_{k=2}^{\infty} f_{2 k}
$$

is proportional to $\left(m_{s}-m_{u d}\right)^{2}$. Therefore, as in Ref.[10], it is convenient to consider a ratio

$$
R_{\Delta f}=\frac{\Delta f}{\left(M_{K}^{2}-M_{\pi}^{2}\right)^{2}}
$$

for the chiral extrapolation of $f_{+}(0)$. In this analysis, $f_{2}$ at the simulated quark masses is calculated by using the ChPT formula in unquenched QCD [7] $]^{1}$.

Our results for $R_{\Delta f}$ are plotted in Fig. 3. Since the single sea quark mass is simulated so far, we extrapolate $R_{\Delta f}$ only in terms of the valence quark mass by naively assuming that its sea quark mass dependence is not large below the simulated sea quark mass. From a simple linear fit shown in Fig. 3, we obtain $f_{+}(0)=0.955(12)$, which is consistent with both of the previous phenomenological estimate 0.961(8) [9] and the quenched result 0.960(9) [10]. We note that recent unquenched calculations in Refs.[11, 12] also obtained similar values for $f_{+}(0)$.

\section{Conclusions}

We have calculated $f_{+}(0)$ in two-flavor QCD using the domain-wall quarks. Our preliminary result is consistent with the previous phenomenological estimate with an accuracy of $1 \%$ level. Our estimate combined with recent experimental results of $\Gamma$ leads to $\left|V_{u s}\right|$ which is consistent

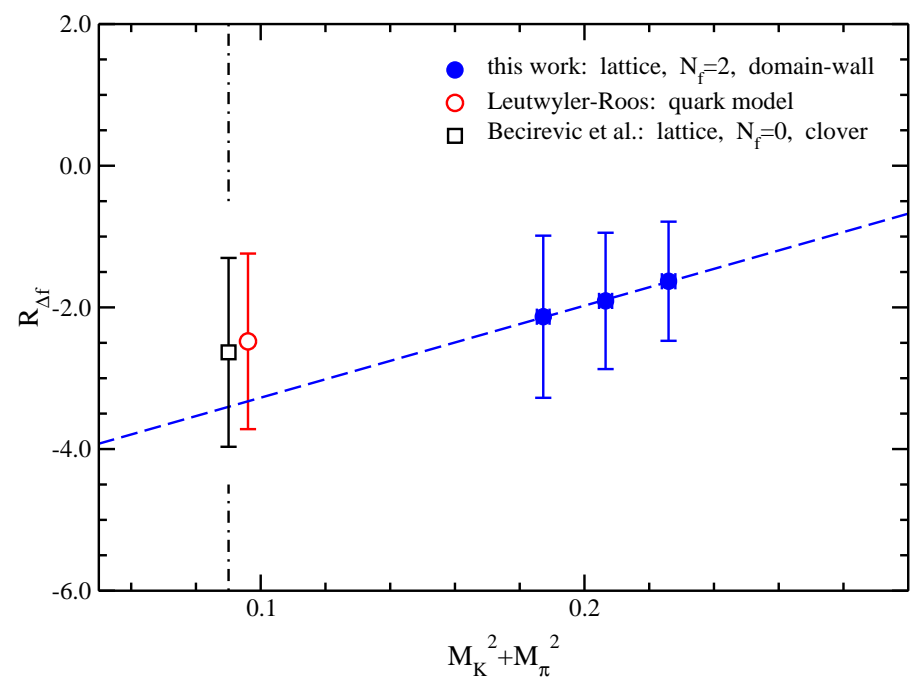

Figure 3: Quark mass dependence of $R_{\Delta f}$. Open circle and square represent values corresponding to the phenomenological estimate [9] and the quenched result [10], respectively.

\footnotetext{
${ }^{1}$ Very recently, $f_{2}$ is calculated in partially quenched ChPT (PQChPT) [17]. We confi rm that the chiral extrapolation with the PQChPT formula for $f_{2}$ leads to small change $(\sim 0.5 \%)$ in $f_{+}(0)$.
} 
with the CKM unitarity. For instance, by using $\Gamma$ from the E865 Collaboration [2], we obtain $\left|V_{u s}\right|=0.229(4)$ which leads to

$$
\left|V_{u d}\right|^{2}+\left|V_{u s}\right|^{2}+\left|V_{u b}\right|^{2}=1-\delta, \quad \delta=-0.001(2) .
$$

We note that, however, our result has an additional uncertainty arising from the fact that we have not taken the limit of the physical sea quark mass. To remove this uncertainty, our simulations at two different sea quark masses are in progress.

The nice consistency between our and previous quenched estimates of $f_{+}(0)$ may suggest that the systematic error due to the quenched approximation for strange quarks is not large. This point, however, has to be confirmed by extending our calculation to three-flavor QCD.

We thank RIKEN, BNL and the U.S. Department of Energy for providing the facilities essential for this work. The work of TK is supported in part by the Grant-in-Aid of the Japanese Ministry of Education (Nos.17740171). The work of AS was supported in part by US DOE Contract No. DE-AC02-98CH10886.

\section{References}

[1] S. Eidelman et al., Phys. Lett. B 592, 1 (2004).

[2] A. Sher et al. (E865 Collaboration), Phys. Rev. Lett. 91, 261802 (2003).

[3] T. Alexopoulos et al. (KTeV Collaboration), Phys. Rev. Lett. 93, 181802 (2004).

[4] A. Lai et al. (NA 48 Collaboration), Phys. Lett. B 602, 41 (2004).

[5] P. Franzini, eConf C0406271 (2004); G. Lanfranchi (KLOE Collaboration), Int. J. Mod. Phys A 20, 493 (2005).

[6] R.E. Behrends and A. Sirlin, Phys. Rev. Lett. 4, 186 (1960); M. Ademollo and R. Gatto, Phys. Rev. Lett. 13, 264 (1964).

[7] J. Gasser and H. Leutwyler, Nucl. Phys. B 250, 517 (1985).

[8] J. Bijnens, G. Colangelo, G. Ecker, Phys. Lett. B 441, 437 (1998); P. Post and K. Schilcher, Eur. Phys. J. C 25, 427 (2002).

[9] H. Leutwyler and M.Roos, Z. Phys. C 25, 91 (1984).

[10] D. Bećirević et al., Nucl. Phys. B 705, 339 (2005); Eur. Phys. J. A24S1, 69 (2005).

[11] N. Tsutsui et al. (JLQCD Collaboration), in these proceedings.

[12] M. Okamoto (Fermilab-MILC-HPQCD Collaborations), hep-lat/0412044.

[13] D.B. Kaplan, Phys. Lett. B 288, 342 (1992); Y. Shamir, Nucl. Phys. B 406, 90 (1993).

[14] T. Takaishi, Phys. Rev. D 54, 1050 (1996); Ph. de Forcrand et al. (QCD-TARO Collaboration), Nucl. Phys. B 577, 263 (2000).

[15] Y. Aoki et al. (RBC Collaboration), hep-lat/0411006.

[16] S. Hashimoto et al., Phys. Rev. D 61, 014502 (2000).

[17] D. Bećirević, G. Martinelli and G. Villadoro, hep-lat/0508013. 